\title{
Prognosis of small hepatocellular carcinoma treated by percutaneous ethanol injection and transcatheter arterial chemoembolization
}

\author{
Chao-Sheng Liao ${ }^{\mathrm{a}}$, Kuo-Ching Yang ${ }^{\mathrm{a}}$, Ming-Fang Yen ${ }^{\mathrm{b}}$, Li-Lian Teng ${ }^{\mathrm{b}}$, Stephen W. Duffy ${ }^{\mathrm{c}}$, \\ Tony Hsiu-Hsi Chen ${ }^{\mathrm{b}, *}$

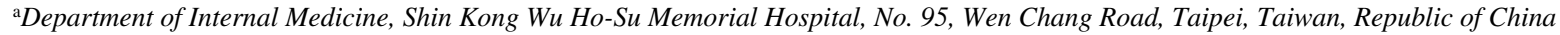 \\ ${ }^{\mathrm{b}}$ Institute of Preventive Medicine, College of Public Health, National Taiwan University, Room 19, Hsuchow Road, Taipei, Taiwan, Republic of China \\ 'Imperial Cancer Research Fund, P.O. Box 123, Lincoln's Inn Fields, London, WC2A 3PX, United Kingdom
}

Received 14 November 2001; received in revised form 20 March 2002; accepted 19 June 2002

\begin{abstract}
This study was conducted to assess the progression and prognosis of a total of 108 patients with hepatocellular carcinoma (HCCs) smaller than 5 $\mathrm{cm}$ in diameter treated by percutaneous ethanol injection (PEI) with or without transcatheter arterial chemoembolization. All patients were classified as Child-Pugh A $(n=84)$ or B $(n=24)$. Logarithm of hazard rate (per month) with time since therapy was assessed. The Weibull model was used to elucidate the effect of pretreatment clinico-pathologic variables on prognosis. The rate of death increased by $4.7 \%$ (95\% CI: 3.7-5.7\%) per month since treatment. Child-Pugh B status was associated with a 2.8-fold risk (95\% CI: 1.52-5.16) of death. Those with a high level of AST or alcoholic cirrhotics had a two-fold risk (95\% CI:1.14-3.42) for death from HCC. Our results suggest the optimal frequency of clinical surveillance of small HCC cases after treatment should take account of increased hazard rate with time and the roles of pretreatment clinico-pathologic variables. (C) 2002 Elsevier Science Inc. All rights reserved.
\end{abstract}

Keywords: Small hepatocellular carcinoma; Percutaneous ethanol injection; Child-Pugh classification; Prognosis; Weibull model

\section{Introduction}

Although the long-term survival rate for patients with small hepatocellular carcinoma (HCCs) treated with percutaneous ethanol injection (PEI) with/without transcatheter arterial embolization or chemoembolization (TAE/TACE) has been reported to be comparable to that of patients undergoing surgical hepatic resection [1-4], prognosis of small HCC treated by PEI has been observed to deteriorate with time after treatment. Table 1 summarizes the estimated hazard rates of death for small HCC treated by PEI with or without TAE/TACE from the previous studies between 1987 and 1997 [2-9]. It can be seen that most studies on PEI show that the annual risk of death from $\mathrm{HCC}$ for patients treated with PEI increases with time. For example, the largest study in Italy [4] shows the rate of death per 1000 per month for a solitary tumor less than $3 \mathrm{~cm}$ increasing from $2.54 / 1000$ per month in the first year to $29.51 / 1000$ per 7707.

* Corresponding author. Tel.: +886-2-2358-7630; fax: +886-2-2358-

E-mail address: stony @episerv.cph.ntu.edu.tw (T.H.H. Chen). month in the fifth year. Similar findings were also observed for tumors with size between 3 and $5 \mathrm{~cm}$ in diameter or tumors with multiple nodules.

By contrast, the hazard rate of HCC by surgical treatment tends to decrease or to remain constant with time [10-14]. For example, Chen et al. [10] reported 56\%, 40\%, 36\%, and 33\% for the 1-, 2-, 3-, and 4-year of survival rates for 205 patients. Hazard rates for HCC cases treated with hepatic resection in Iwatsuki and Starzl [12] decrease from 54.57/1000 per month in the first three months to $12.81 / 1000$ in the fifth year.

Regarding the overall prognosis of small HCC cases treated by PEI, previous studies showed cumulative survival or hazard rate of patients with PEI varies and is probably dependent on factors pertaining to prognosis of HCC, for example, Child-Pugh classification and tumor size. This implies that prediction of prognosis for small HCC should take relevant covariates into account. It would be very informative to identify pretreatment variables predictive of outcome, while taking the nonconstant hazard rate mentioned above into account.

To further quantify this phenomenon, we applied a simple survival method shown in the Appendix A to estimate the 
Table 1

Estimated hazard rates of death for patients with HCC treated by PEI, from the literature between 1995 and 2000

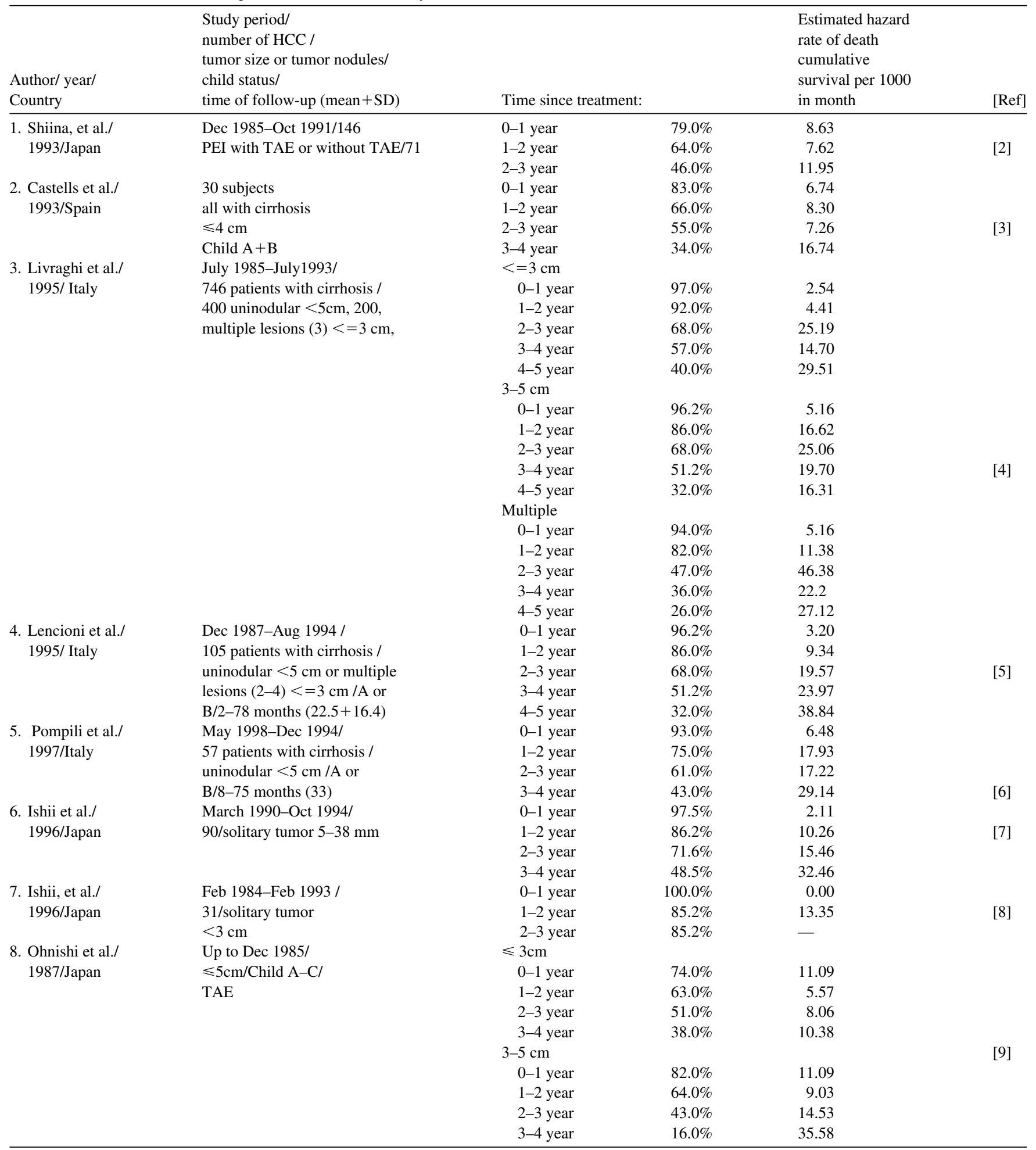

Details of calculation of hazard rate are given in Appendix A.

hazard rate of death per month of follow-up after systematic literature reviews. The aims of this study are therefore to examine whether the increased hazard rate of death for small HCC cases treated by PEI is also observed in Taiwanese pa- tients using a time trend equation. Taking non-constant rate of death into account, analysis of prognosis of $\mathrm{HCC}$ was performed by the use of the Weibull model. Finally, a predictive Weibull model for HCC treated with PEI was developed to 
provide information for the clinical physician to choose the optimal treatment for patients with small HCC.

\section{Methods}

\subsection{Patients}

Between January 1991 and December 1999, a total of 108 patients with HCCs smaller than $5 \mathrm{~cm}$ in diameter treated by PEI with or without TAE/TACE at one large regional hospital in Taipei, Taiwan, were recruited under the approval by ethics committee. All patients were unsuitable for surgical hepatic resection because of liver dysfunction, presence of lesions in locations that not amenable to hepatic resection, or coexistence of other disease. All patients had liver cirrhosis. They were confirmed histologically in 48 patients. For the remaining 60 patients, cirrhotic liver were diagnosed by unequivocal clinical criteria, that is, coarse liver parenchyma with uneven or nodular surface on images of ultrasonography (US) and computed tomography (CT), corkscrew vascular pattern on hepatic angiography, presence of portal hypertension (esophageal or gastric varices, enlarged spleen, reopening ductus umbilicus) in chronic liver disease patients. Criteria for treatment with PEI included: (1) single, nodular HCC smaller than $5 \mathrm{~cm}$ or multiple (up to four) nodular HCC lesions less than $5 \mathrm{~cm}$ each; (2) no portal vein thrombosis or extrahepatic metastases; (3) cirrhosis classified as Child-Pugh A or B; (4) absence of any symptoms related to bleeding and prothrombin time less than $16 \mathrm{sec}$ and platelet count higher than $40,000 / \mu \mathrm{L}$. The number of tumor nodules and absence of portal vein thrombosis were confirmed on the basis of US and CT scan findings. Tumor size in diameter was rated by US. Extrahepatic metastases was investigated by means of clinical assessment, chest X-ray, abdominal US, and CT.

The patients were 38 females and 70 males with an average of 61 years ( \pm S.D., \pm 9.4 years). A total of 59 deaths from HCC were ascertained after follow-up until 31, July 2000.The median follow-up time was approximately 40 months. Characteristics of patients are listed in Table 2 . Hepatitis B surface antigen (HBsAg) was positive in 48 patients, and hepatitis $\mathrm{C}$ virus (HCV) antibody was positive in 51. None had both hepatitis B surface antigen and HCV antibody. Ninety-seven patients had a uninodular tumor, and only 11 had a multinodular tumor (at least four nodules). The size of the treated tumors ranged between 0.7 and 4.9 $\mathrm{cm}$ with mean value, $2.39 \mathrm{~cm}( \pm$ S.D., \pm 1.06$)$. PEI was the first choice of treatment for uninodular tumors less than 3 $\mathrm{cm}$ in diameter. Combined PEI with TAE/TACE therapy was undertaken to HCCs between 3 and $5 \mathrm{~cm}$ in diameter. The guideline for the combined therapy is that when angiography indicated a hypervascular tumor and liver function was preserved enough for TAE/TACE, TAE/TACE was performed with PEI therapy. In this study, 64 of 108 patients $(59 \%)$ were treated with PEI in combination with TAE/TACE. Before treatment, all patients were examined by US and dynamic CT with intravenous contrast enhancement. The diagnosis of HCC was confirmed by (1) USguided fine-needle biopsy of the lesion in 35 patients; (2) focal hepatic nodule(s) with typical images of HCC on US, $\mathrm{CT}$, angiography combined with elevated $\alpha$-fetoprotein (AFP) level above $200 \mathrm{ng} / \mathrm{mL}$ in 21 patients; (3) focal hepatic nodule(s) with typical images of HCC on US and CT combined with hypervascular tumor stains on angiography in 46 patients; (4) focal hepatic nodule(s) without typical images of $\mathrm{HCC}$ on US, CT, or angiography presenting with progression of tumor in six patients. Thirty of the 108 (27.8\%) patients were found to have elevated AFP level above $200 \mathrm{ng} / \mathrm{mL}$. After the PEI cycle, patients were examined by US and CT at 1-month intervals. Levels of AFP were also measured. If a viable tumor was found the administration of PEI was repeated. If no residual tumor was found, the patients were subjected to periodical monitoring with AFP measurement and sonography at 3-month interval and CT scan at 6-month intervals.

\subsection{Prognostic factors}

The pretreatment clinico-pathologic variables evaluated for an effect on survival in this study include patient- and tumor-related variables. Patient-related factors were sex, age, causes of cirrhosis (indicated by serology test of HBsAg, anti-HCV, and habit of alcohol consumption), severity of liver dysfunction (Class-Pugh A or B), serum albumin level, serum total bilirubin level, serum aspartate transaminase (AST), and alanine transaminase (ALT) levels, prothrombin time, and presence of ascites. Tumor-related variables include types of tumor (solitary or multiple nodules) and diameters of tumor. The levels of AST and ALT were dichotomized according to the median values (56 IU/L for AST, and $40 \mathrm{IU} / \mathrm{L}$ for ALT). Serum AFP level above 200 $\mathrm{ng} / \mathrm{mL}$ was classified as high AFP group.

\subsection{Statistical methods}

\subsubsection{Time trend equations}

To assess whether the risk of death increased with time, time trend equations were derived by following procedure:

1. Cumulative survival rates of HCC cases were first analyzed by the Kaplan-Meier method. The differences of cumulative survival were assessed by the use of Log-rank method. .

2. The hazard rate per month was calculated on the basis of the fundamental concept of survival analysis [15]. This is described in Appendix A.

3. A time trend equation was applied to regress the logarithm of hazard rate (per month) on time since therapy.

$$
\operatorname{Ln}(h(t))=\beta t+\alpha
$$

where $\beta$ and $\alpha$ are slope and intercept of logarithm of hazard rate.

4. To estimate stratum-specific hazard rates in relation to prognostic factors such as Child-Pugh status, a re- 
Table 2

Cumulative survival of small HCC cases by demographic and clinico-pathologic variables

\begin{tabular}{|c|c|c|c|c|c|c|}
\hline \multirow[b]{2}{*}{ Variable } & \multirow{2}{*}{$\begin{array}{l}\text { No. of deaths / } \\
\text { No. of patients }\end{array}$} & \multicolumn{3}{|c|}{ Cumulative survival } & \multirow[b]{2}{*}{$\chi^{2}$} & \multirow[b]{2}{*}{$\mathrm{P}$-value } \\
\hline & & 1-Year & 3-Year & $\overline{5-Y e a r}$ & & \\
\hline Sex & & & & & 3.24 & 0.0718 \\
\hline Male & $42 / 70$ & 0.8857 & 0.4827 & 0.0955 & & \\
\hline Female & $17 / 38$ & 0.9737 & 0.6510 & 0.4780 & & \\
\hline Age & & & & & 1.39 & 0.5000 \\
\hline$<55$ & $13 / 29$ & 0.9655 & 0.6123 & 0.3108 & & \\
\hline $55-64$ & $25 / 44$ & 0.8864 & 0.5765 & 0.1625 & & \\
\hline Hepatitis $^{\mathrm{a}}$ & $49 / 95$ & 0.9158 & 0.5905 & 0.2623 & & \\
\hline Alcohol & $10 / 13$ & 0.9231 & 0.1579 & 0.1579 & & \\
\hline HBsAg & & & & & 0.07 & 0.7914 \\
\hline Positive & $27 / 48$ & 0.8958 & 0.5659 & 0.2388 & & \\
\hline Negative & $32 / 60$ & 0.9333 & 0.5117 & 0.2467 & & \\
\hline Anti-HCV & & & & & 1.00 & 0.3185 \\
\hline Child-Pugh B & $21 / 24$ & 0.7500 & 0.1893 & 0.0631 & & \\
\hline Level of AFP & & & & & 0.0907 & 0.7633 \\
\hline$<200 \mathrm{ng} / \mathrm{mL}$ & $41 / 78$ & 0.9359 & 0.5709 & 0.2336 & & \\
\hline$\geqslant 200 \mathrm{ng} / \mathrm{mL}$ & $18 / 30$ & 0.8667 & 0.4551 & 0.2600 & & \\
\hline Type of tumor & & & & & 7.05 & 0.0079 \\
\hline Solitary & $50 / 97$ & 0.9278 & 0.5486 & 0.2709 & & \\
\hline Multiple & $9 / 11$ & 0.8182 & 0.4242 & 0.1414 & & \\
\hline Tumor size & & & & & 1.50 & 0.2212 \\
\hline$<2 \mathrm{~cm}$ & $15 / 33$ & 0.9091 & 0.6208 & 0.4997 & & \\
\hline $2 \sim 5 \mathrm{~cm}$ & $35 / 64$ & 0.9375 & 0.5027 & 0.1496 & & \\
\hline Level of AST & & & & & 7.76 & 0.0053 \\
\hline Low $(\leqslant 56$ IU/L) & $24 / 54$ & 0.9630 & 0.6543 & 0.3852 & & \\
\hline $\operatorname{High}(>56 \mathrm{IU} / \mathrm{L})$ & $35 / 54$ & 0.8704 & 0.4070 & 0.1135 & & \\
\hline
\end{tabular}

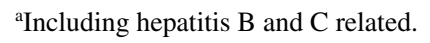

gression model with interaction term between time and certain specific prognostic factor was used. Take Child-Pugh status (denoted as $X_{4}$ ), for example, the regression model with the interaction term $\left(X_{4} \times t\right)$ was expressed as:

$$
\operatorname{Ln}(h(t))=\beta_{1} \times t+\beta_{2} \times X_{4} \times t+\alpha
$$

Note that Child-Pugh A and Child-Pugh B are denoted as $X_{4}=0$ and $X_{4}=1$, respectively. Two regression coefficients, $\beta_{1}$ and $\beta_{2}$, are slopes of logarithm of hazard rate for Child-Pugh A and Child-Pugh B, respectively.

\subsubsection{The Weibull model}

Because it was postulated that the hazard rate of death from HCC increases with time of follow-up, multiple regression using the Weibull model was applied to identify significant prognostic factors. The Weibull model approach is a parameteric survival model for accommodating hazard rates at different times. Clinical application to HCC cases is illustrated in Appendix B. This model was further used to predict the absolute survival of HCC cases treated by PEI based on clinico-pathologic variables. The detailed algebra for survival probability for the Weibull model is also given in Appendix B.

The predicted cumulative survival by Weibull model was compared with the observed cumulative survival curve calculated by life-table method. Goodness of fit test between two curves by 3-month interval was also assessed.

\section{Results}

\subsection{Cumulative survival and hazard rate}

The overall survival rates of HCC treated by PEI and PEI in combination with TAE/TACE for 1-5 years were estimated as $92 \%, 72 \%, 54 \%, 34 \%$, and $24 \%$, respectively. The hazard rate of death increased with time (Fig. 1). The increasing trend expressed by the slope of 0.0460 ( $\mathrm{SE}=0.005)$ us- 
Table 3

Hazard ratios from the Weibull regression model

\begin{tabular}{|c|c|c|c|c|}
\hline Variable & $\begin{array}{l}\text { Weight } \\
\text { (regression } \\
\text { coefficient) }\end{array}$ & $\begin{array}{l}\text { Hazard } \\
\text { rate }\end{array}$ & $95 \% \mathrm{CI}$ & P-value \\
\hline Age $\left(X_{1}\right)$ & -0.01 & 1.02 & $0.99-1.04$ & 0.2635 \\
\hline $\operatorname{Sex}\left(X_{2}\right)$ & & & & 0.0845 \\
\hline Male (=1) & -0.30 & 1.74 & $0.93-3.27$ & \\
\hline Female $(=0)$ & & 1.00 & - & \\
\hline Treat modality $\left(\mathrm{X}_{3}\right)$ & & & & 0.4081 \\
\hline PEI $(=1)$ & 0.14 & 0.77 & $0.41-1.44$ & \\
\hline PEI+TAE/TACE $(=0)$ & & 1.00 & - & \\
\hline Liver dysfunction $\left(\mathrm{X}_{4}\right)$ & & & & 0.0012 \\
\hline Child-Pugh B $(=1)$ & -0.56 & 2.80 & $1.52-5.16$ & \\
\hline Child-Pugh A $(=0)$ & & 1.00 & & \\
\hline Cause of cirrhosis $\left(\mathrm{X}_{5}\right)$ & & & & 0.0455 \\
\hline Alcohol (=1) & -0.41 & 2.12 & $1.01-4.48$ & \\
\hline Hepatitis $(=0)$ & & 1.00 & - & \\
\hline Level of AFP $\left(\mathrm{X}_{6}\right)$ & & & & 0.8191 \\
\hline$>200 \mathrm{ng} / \mathrm{mL}(=1)$ & -0.04 & 1.07 & $0.59-1.94$ & \\
\hline$\leqslant 200 \mathrm{ng} / \mathrm{mL}(=0)$ & & 1.00 & - & \\
\hline Level of AST $\left(\mathrm{X}_{7}\right)$ & & & & 0.0158 \\
\hline $\operatorname{High}(=1)$ & -0.37 & 1.97 & $1.14-3.42$ & \\
\hline Low $(=0)$ & & 1.00 & & \\
\hline \multicolumn{5}{|l|}{ Tumor size $\left(\mathrm{X}_{8}\right.$ or $\left.\mathrm{X}_{9}\right)$} \\
\hline$\leqslant 2 \mathrm{~cm}\left(\mathrm{X}_{8}=0, \mathrm{X}_{9}=0\right)$ & & 1.00 & - & \\
\hline Single, $2-5 \mathrm{~cm}\left(\mathrm{X}_{8}=1, \mathrm{X}_{9}=0\right)$ & -0.03 & 1.06 & $0.53-2.14$ & 0.8654 \\
\hline Multiple $\left(\mathrm{X}_{8}=0, \mathrm{X}_{9}=1\right)$ & -0.28 & 1.68 & $0.62-4.54$ & 0.3079 \\
\hline Scale parameter $(\sigma)$ & 0.54 & & & \\
\hline
\end{tabular}

ing the time trend equation from expression (1) reaches statistical significance $(t=9.10, \mathrm{P}<.0001)$. This corresponds to a $4.7 \%$ (95\% CI: $3.7-5.7 \%$ ) increase per month in the hazard rate. Similar linear trends were also found for PEI without TAE/TACE [4.79\% (95\% CI: $3.87-5.72 \%$ ) per month] and for PEI with TAE/TACE $[5.25 \%$ (95\% CI: 4.46-6.04\%) per month].

Table 2 shows 1-year, 3-year, and 5-year cumulative survival by relevant clinico-pathologic characteristics before therapy. Only etiology of liver cirrhosis, Child-Pugh status, type of tumor, and AST show statistical significance in accounting for prognosis of small HCC cases.

Males have poorer survival than females. The difference is of borderline statistical significance $\left(\chi_{(1)}^{2}=3.24, \mathrm{P}=\right.$ .0718). The hazard rate increase was only slightly higher in males [4.83\% (95\% CI: 3.44-6.23\%) per month] than in females [4.25\% (95\% CI: $2.77-5.75 \%$ ) per month].

Cumulative survival rates for Child-Pugh $\mathrm{A}$ at 1 year, 2 years, 3 years, 4 years, and 5 years were estimated as $96.43 \%, 80.16 \%, 63.60 \%, 42.91 \%$, and $32.91 \%$, respectively. One-year, 2-year, 3-year, and 4-year survival rates for Child-Pugh B are 75, 44.62, 18.93, and 6.31\%, respectively. There is a significant association between Child status and death $\left(\chi_{(1)}^{2}=28.59, \mathrm{P}<.0001\right)$. Figure 2 shows that the hazard rate of death for Child-Pugh $B$ accelerates more remarkably with time than that of Child-Pugh A. The respective trend equations give $4.21 \%$ (95\%CI: $2.98-5.46 \%$ ) per month and $10.02 \%$ (95\% CI: 7.95-12.12\%) per month increases in the hazard rate for Child-Pugh A and Child-Pugh $\mathrm{B}$, respectively.

HCC cases with multiple nodules had poorer survival rate than those with solitary tumor $\left(\chi_{(1)}^{2}=8.49, \mathrm{P}=.014\right)$. However, there was no substantial difference between tumors less than $2 \mathrm{~cm}$ and tumors between 2 and $5 \mathrm{~cm}$. Consequently, the hazard rate of death for multiple nodules grows faster with time than that for solitary tumors (Fig. 3). Time trend equations yielded a $10.22 \%(95 \%$ CI: $7.80-12.91 \%)$ increase in the death hazard rate per month for those with multiple nodules, a $4.41 \%$ (95\% CI: $3.07-5.77 \%$ ) increase for tumors of size $2-5 \mathrm{~cm}$, and a $3.96 \%$ (95\% CI: $2.12-5.82 \%$ ) increase in those with solitary tumors smaller than $2 \mathrm{~cm}$.

Although the 1-year survival associated with high levels of AST was similar to that of low levels (87\% vs. 96\%), there were substantial absolute benefit differences in 3-year ( $41 \%$ vs. $65 \%)$ and 5 -year (11\% vs. $39 \%$ ) survival. Overall, those with high levels had significantly poorer survival $(\mathrm{P}=$

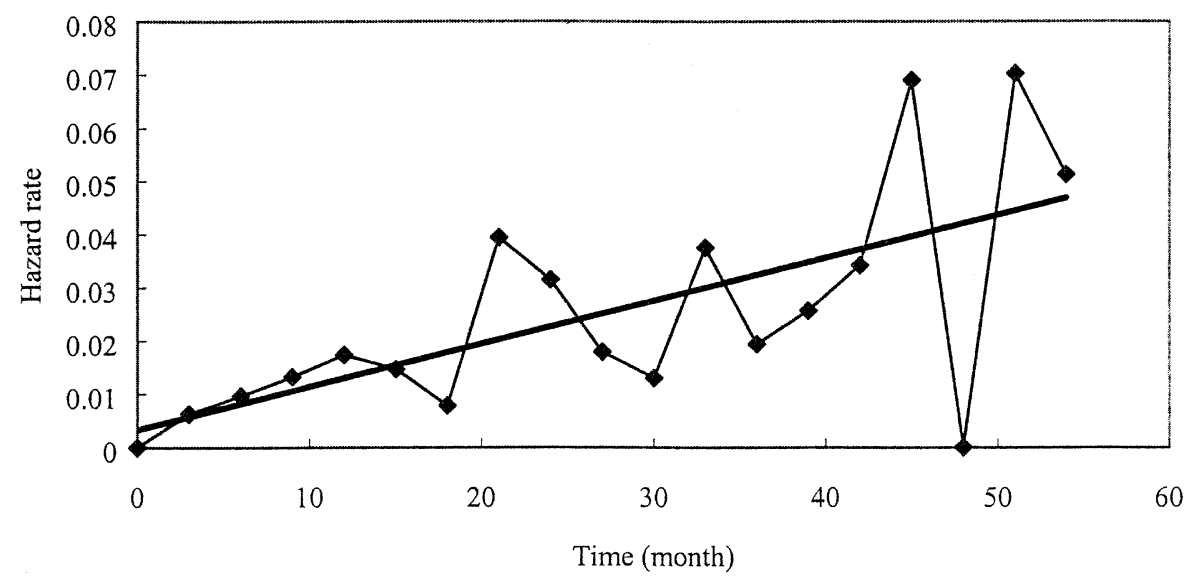

Fig. 1. The hazard rate of death for small HCC cases treated by PEI with or without TAE. 


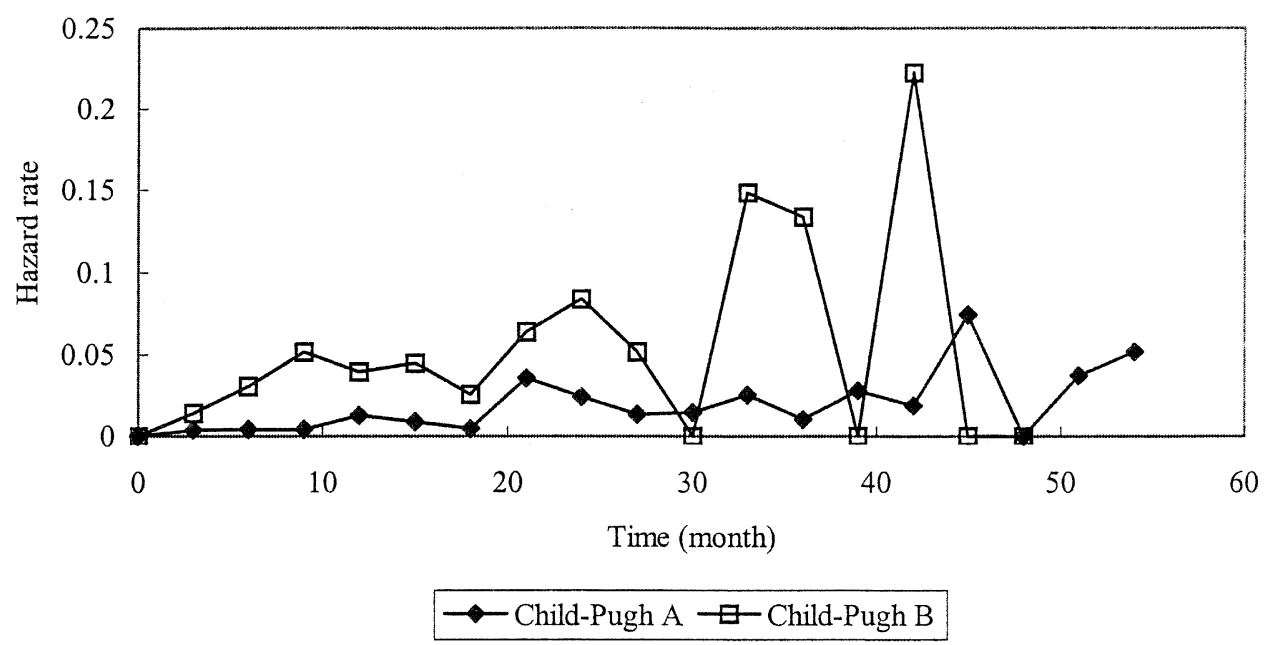

Fig. 2. The hazard rate of death for small HCC cases treated by PEI stratified by Child-Pugh class (A/B).

$.005)$. Figure 4 shows the hazard rate by time and AST levels. In addition to being greater on average in those with high levels of AST, the rate of death also increases more rapidly with time in this group, varying by $5.7 \%$ (95\% CI: 4.14-7.29\%) per month compared to $4.52 \%$ (95\% CI: $3.28-$ $5.77 \%$ ) per month in those with low levels.

\subsection{Multiple regression using the Weibull model}

Fitting the survival time with the Weibull model without covariates yields the scale parameter equal to 0.65 . Taking this into account, results of the Weibull regression model are shown in Table 3. Child-Pugh status, etiology of cirrhosis, and AST were still highly associated with the prognosis of HCC after adjustment for other variables. Males are 1.74 times (95\% CI: $0.93-3.28)$ likely to die from HCC as females, with borderline statistical significance $\left(\chi_{(1)}^{2}=2.98\right.$, $\mathrm{P}=.085$ ). Child-Pugh B has a 2.8-fold (95\% CI: 1.52-5.16) risk for death from HCC compared with Child-Pugh A $\left(\chi_{(1)}^{2}=10.48, \mathrm{P}=.0012\right)$. Those with high level of AST have a two-fold (95\% CI:1.14-3.42) risk for death from HCC compared with those with low level of AST $\left(\chi_{(1)}^{2}=\right.$ $5.83, \mathrm{P}=.016)$. The scale parameter after adjustment for covariates is 0.54 . This suggests that these covariates may play a key role in the increased risk of death with time.

\subsection{The Weibull model for prediction of survival for HCC cases treated with PEI}

To predict the prognosis of small HCC treated by PEI using pretreatment variables as in the above Weibull regression model, cumulative survival rates were predicted using the Weibull model with covariates including age $\left(x_{1}\right)$, gender $\left(x_{2}\right)$, treatment modality $\left(x_{3}\right)$, Child-Pugh class $\left(x_{4}\right)$, cause of cirrhosis $\left(x_{5}\right)$, level of $\operatorname{AFP}\left(x_{6}\right)$, level of AST $\left(x_{7}\right)$, size of tumor $\left(x_{8}\right)$ or type of tumor $\left(x_{9}\right)$. Table 3 shows each

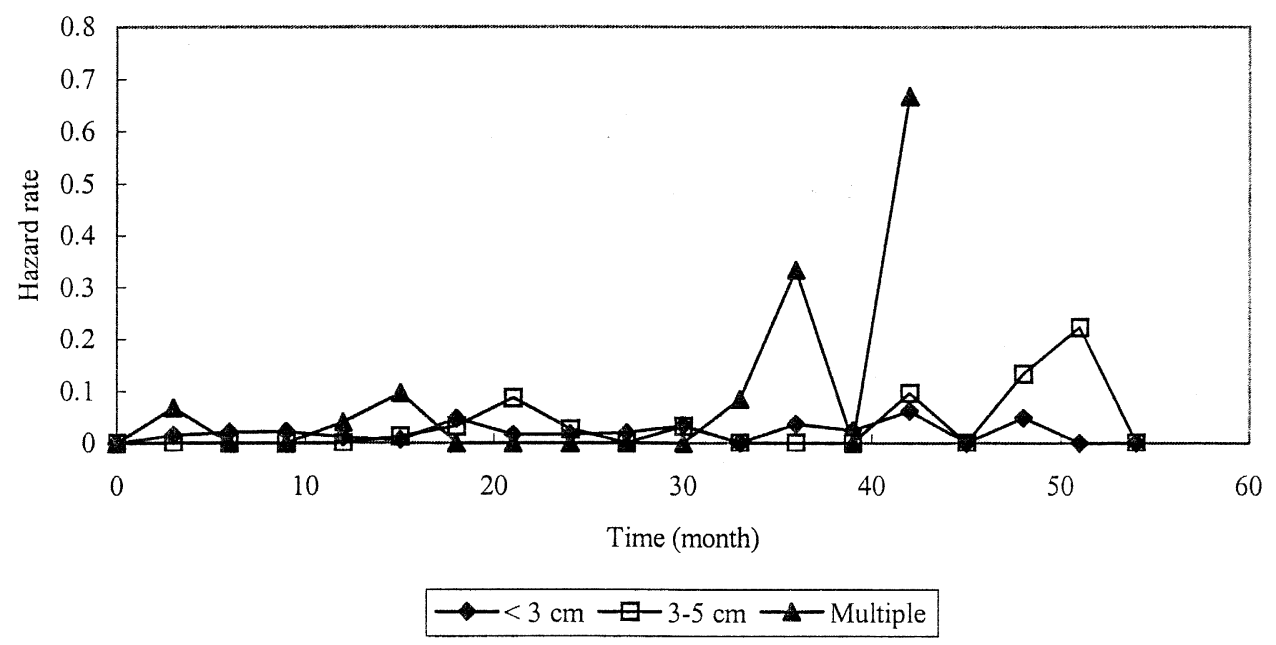

Fig. 3. The hazard rate of death for small HCC cases treated by types of tumor. 


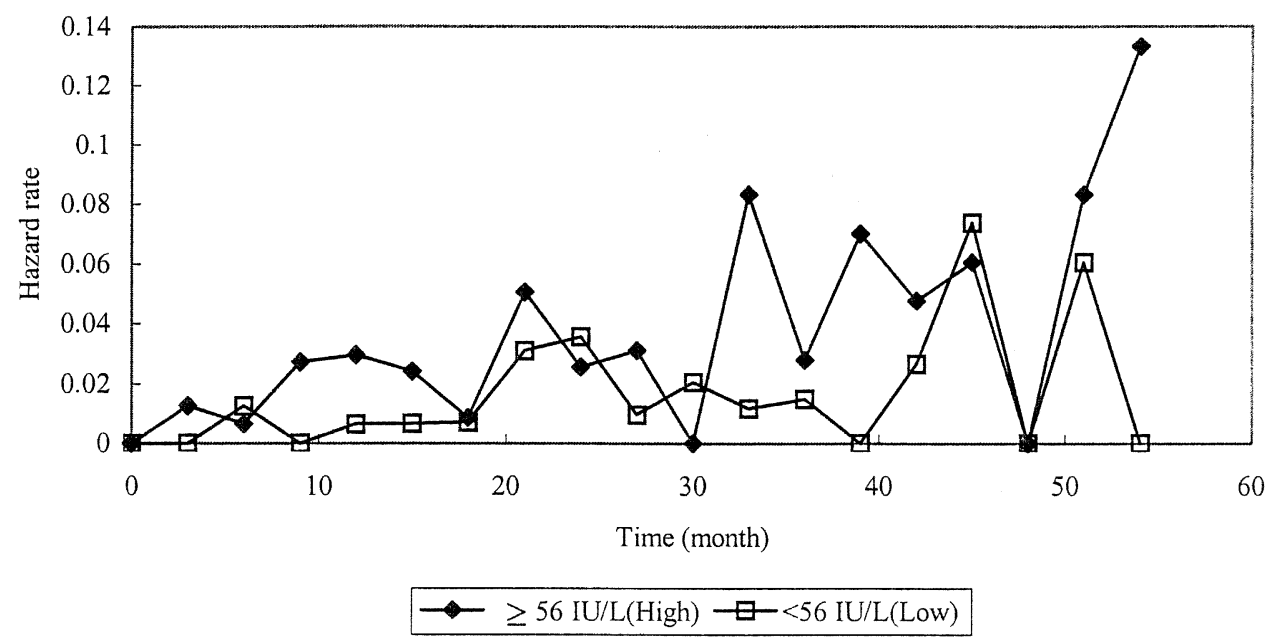

Fig. 4. The hazard rate of death for small HCC cases treated by level of AST.

variable weight for calculating the risk score, $R$, according to the expression (A-2). $R$ is expressed as:

$$
R=4.84-0.0086 x_{1}-0.30 x_{2}-\ldots 0.03 x_{8}-0.28 x_{9}(2)
$$

The scale parameter $(\sigma)$ is estimated as 0.54 .

Thus, the risk score for a male aged 57 years, Child-Pugh A, viral hepatitis, level of AFP less than $200 \mathrm{mg} / \mathrm{ml}$, high level of AST, and multinodular tumor treated by PEI only is calculated as 3.41.

Substituting 3.41 into the expression (A-3) yields the predicted 1-year cumulative survival ( $t=12$ months) given the scale parameter equal to 0.54 for this subject as

$$
\mathrm{S}(12)=\exp \left\{-\left[12 \times \mathrm{e}^{-3.41}\right]^{1 / 0.54}\right\}=83.50 \%
$$

Similarly, the 2- and 3-year cumulative survival rates were predicted as $52.10 \%$ and $25.10 \%$.

Figure 5 shows the predicted survival curved by Weibull model and the cumulative survival curve by life-table method. As goodness of fit test between the two curves lacks of statistical significance $\left(\chi_{(1)}^{2}=18, \mathrm{P}=.84\right)$, this suggests that Weibull model has a good predicted validity.

\section{Comment}

\subsection{Implication for the surveillance of small HCC cases treated by PEI}

The present study found that the risk of death for small HCC cases treated by PEI increases with time among Taiwanese people. The rate of death for small HCC cases treated by PEI increases by $4.7 \%$ (95\% CI: $3.7-5.7 \%$ ) per month. The Weibull regression model found the survival of HCCs treated by PEI was highly dependent on the pretreatment clinico-pathologic variables. This suggests that the optimal frequency of US and CT scan for the surveillance of small HCC cases treated by PEI is not only varied with time

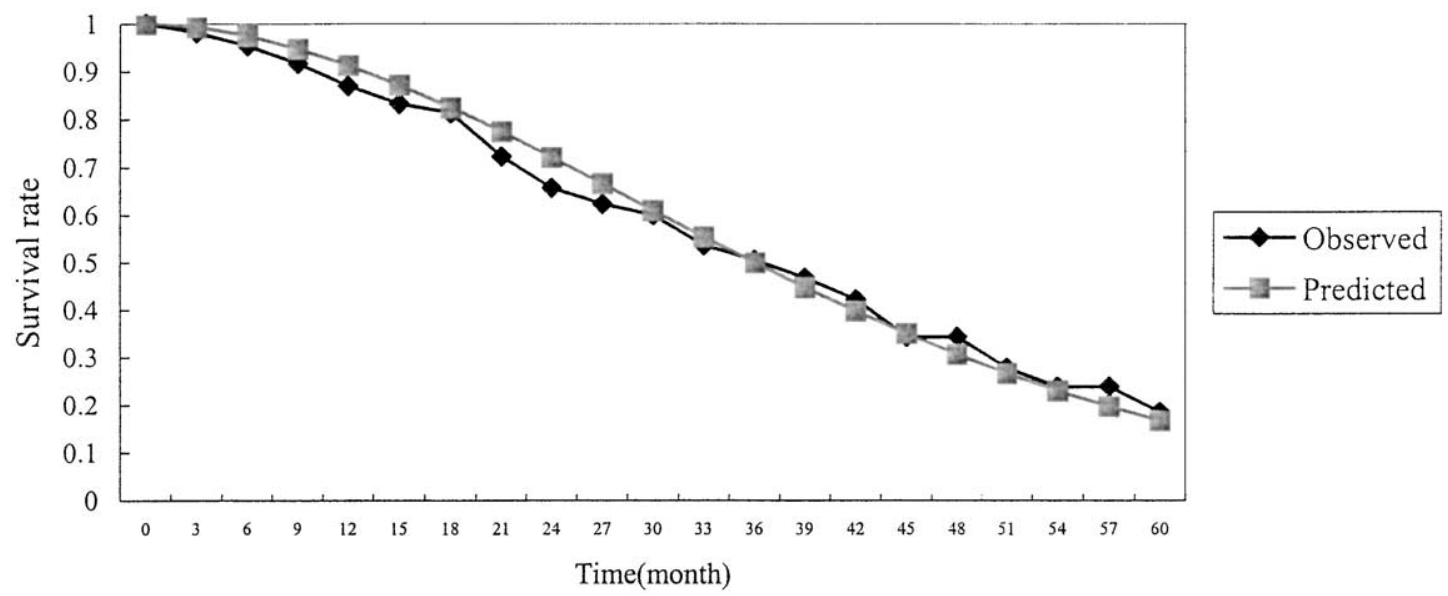

Fig. 5. The predicted cumulative survival curve by the Weibull model and the observed cumulative survival curve by the life-table method. 
but also needs to take these pretreatment clinico-pathologicalal variables into account.

\subsection{Comparisons between current study and earlier findings}

The above increasing trend is also observed in earlier studies, addressing the prognosis of small HCC cases after systematic review. The accelerated rates of risk of death per month after applying the time trend to the logarithm of the hazard rate as above to each study are calculated as $5.01 \%$ in the Lencioni et al. study [5], 3.80\% in the Pompili et al. study [6], 7.44\% in the Ishii et al. study [7], 1.37\% in Shiina et al. [1], 2.19\% in Castells et al. [2], and 3.65\% in the Ohnishi et al. study [10].

\subsection{Prognostic factors}

Prognostic factors for small HCCs are related to the extent to which hazard rate increases with time. The hazard rates with Child-Pugh B, multinodular tumor, and high levels of AST tend to increase faster than those with ChildPugh A, uninodoular, and low levels of AST. Rates of death for small HCC cases grow by $4.21 \%$ (95\% CI: 2.98-5.46\%) and $10.02 \%$ (95\% CI: 7.95-12.12\%) for Child-Pugh A and Child-Pugh B, respectively. The corresponding figures for multinodular, solitary tumor smaller than $3 \mathrm{~cm}$ and solitary tumor between 3 and $5 \mathrm{~cm}$ were $10.22 \%$ (95\% CI: 7.80$12.91 \%$ ), $4.41 \%$ (95\% CI: $3.07-5.77 \%$ ), and $3.96 \%$ (95\% CI: $2.12-5.82 \%$ ), respectively. The accelerated risk of death per month for patients with high levels of AST [5.7\% (95\% CI: 4.14-7.29\%)] is higher than that for patients with low level [4.52\% (95\% CI: 3.28-5.77)]. The difference by size of tumor is at odds with earlier findings $[4,10]$. For example, application of the time trend equation to data from the Ohnishi et al. study gives estimates of increasing hazard of 0.1 and $3.4 \%$ for tumors smaller than or equal to $3 \mathrm{~cm}$ and tumors between 3 and $5 \mathrm{~cm}$, respectively. A similar finding was also observed in Livraghi et al. [4]. The hazard rate for tumor size between 3 and $5 \mathrm{~cm}$ in our study is similar to that in two studies, whereas the corresponding figure for tumor size smaller than $3 \mathrm{~cm}$ is higher than that.

From a clinical viewpoint, an increasing hazard, in particular for large nodule, may suggest that large nodules make a homogeneous ethanol diffusion throughout the entire lesion more difficult for large nodules than for small, due to the texture of the tumor and the intratumoral septa. This can be supported by two earlier findings. First, viable neoplastic tissue along the edge of nodules may remain after treatment. Second, the chance of tumor spread in the vicinity of the lesion is proportional to the size of the tumor. The increasing hazard rates with respect to liver dysfunction (Child-Pugh class), and levels of AST suggest that the occurrence of new lesions after PEI in Child-Pugh B or high level of AST is more likely than that in Child-Pugh A or low levels of AST. To reduce recurrence either from remaining neoplastic tissues or from new lesions, intensive surveillance for these HCCs treated by PEI may be required.
It should be very cautious that remarkable elevation of AFP in hepatitis patients might be due to bridging necrosis of the liver and may not simply imply existence or progression of tumors. However, this may be unlikely in our case because no remarkable exacerbation of hepatitis was found at diagnosis of patients in this series. Furthermore, the correlations between AFP and liver enzymes $[-0.00581$ and 0.02543 for AST and ALT, respectively] also suggest a lack of interdependence between elevations of AFP and elevations of liver enzymes. The follow-up schedule for HCCs treated by PEI is AFP measurement and US at 3-month intervals and CT scanning at 6-month intervals. Whether a uniform schedule of follow-up is appropriate for each individual should be elucidated in the future work.

Chronic viral hepatitis B and C have long been recognized as risk factors of HCC [16,17]. In our series, the prognosis of treated small HCC with respect to the etiology of liver cirrhosis was evaluated. Viral-related cirrhotic patients showed longer survival $(\mathrm{P}=.0063)$. However, the misclassification of etiology of liver cirrhosis should be taken into account when detailed virus information was lacking. Those with negative hepatitis B surface antigen may be antihepatitis B core antibody positive. The recent study conducted by Okada et al. found the elevated risk in patients with negative HBsAg and positive anti-HBc. They also pointed out that the HCC patients with negative HBsAg also showed positive anti-HBc [18].

\subsection{Methodologic consideration}

To enlarge sample size and to make the sample as representative as possible, it is customary to use a multicenter design. There are two reasons for selecting only one hospital for the current study. First, PEI or PEI with TAE/TACE has been the sole treatment method in this hospital. HCC cases to be treated with surgery were referred to other medical centers. Therefore, guidelines for selecting patients treated by PEI are predetermined rather than arbitrary. Second, treatment decisions and criteria are rather diversified in the majority of hospitals in Taiwan, it is therefore difficult to identify a homogeneous HCC cohort treated with PEI on the basis of a multicenter design, albeit multicenter design can increase sample sizes.

The Weibull model proposed in the current study is simple and useful for the prediction of cumulative survival given pretreatment variables, taking the property of increasing hazard into account, and incorporating the effects of a series of relevant covariates. In this model, we used ChildPugh stage as the single liver function variable to reduce heterogeneity. One may suggest adopting a series of variables for Child-Pugh staging rather than a single index. However, after comparing the results between both models (data not shown), there is no substantial difference. The scale parameter in relation to nonconstant hazard rate was incorporated to calculate predicted cumulative survival as in the expression (A-3). This model can help the clinical physicians with information on prognosis for patients with 
small HCC cases before treatment. More importantly, as single HCC legion with a diameter less or equal to $3 \mathrm{~cm}$ that usually benefits much more than larger tumor has shown a 3\% increasing hazard, an intensive surveillance strategy for such small tumors cannot be overemphasized. However, due to small sample size and the lack of external data, validation of this model is not performed in the current study. It is hoped that if this model is validated in the future, a computer-aided system with the incorporation of the Weibull model can be developed to help the clinical physicians. Similar research for other treatment modalities would provide a valuable aid for therapeutic decision making.

In conclusion, the above findings suggest the optimal frequency of US and CT scan for the surveillance of small HCC cases treated by PEI should take account of increased hazard rate with time and the roles of pretreatment clinicopathologic variables.

\section{Appendix A-calculation of hazard rate}

We calculated the estimated hazard rate on the basis of the concept of nonparametric survival analysis [15]. Let cumulative survival at $x$ years be denoted by $S_{x}$. $S_{x}$ is an unconditional probability of being alive $x$ years after diagnosis. $S_{x}$ can be expressed by the probability of survival, say $T$, $P(T \geqslant x)$. To calculate the hazard rate at different years or months we have to estimate the conditional probability of survival, say $C_{x}=P(T \geqslant x \mid T \geq x-1)$, i.e., the probability of remaining alive between $x-1$ and $x$ years given survival to $x-1$ years. The definition of probability yields the relationship between $S_{x}$ and $C_{x}$ :

$$
\mathrm{C}_{\mathrm{x}}=\mathrm{S}_{\mathrm{x}} / \mathrm{S}_{\mathrm{x}-1} .
$$

Let the hazard rate be denoted by $\mathrm{h}$ and assume that survival time within 1 year follows the exponential distribution. The hazard rate (per 1000 in month), $h$, can then be estimated by:

$$
h=-\operatorname{Ln}\left(C_{x}\right) / 12 .
$$

For example, in Lencioni et al. [5], $C_{x}$ at the second year was estimated as $0.86 / 0.962=0.894$. Thus, the estimate of $h$ at the second year was 9.34 (per 1000 in a month).

\section{Appendix B-Weibull model of survival with changing hazard rate with time}

Let the survival time of HCC cases since time treated by PEI be denoted as $T$ and $k$ relevant clinico-pathologic variables be denoted by $x_{1}, \ldots x_{k}$. The relationship between $T$ and relevant clinico-pathologic variables, according to the log-linear form of the accelerated failure time model (AFT) following Allison [19], is expressed as:

$$
\operatorname{Ln} T_{i}=\beta_{0}+\beta_{1} x_{i 1}+\ldots+\beta_{k} x_{i k}+\sigma \varepsilon_{i}
$$

where $\varepsilon_{i}$ is a random disturbance term, and $\beta_{0}, \beta_{1}, \ldots, \beta_{k}$ are regression coefficients for clinico-pathologic variables and $\sigma$ is shape parameter for different types of survival time distribution.

It should be noted that if the variance of $\varepsilon$ is fixed as standard value (e.g., 1.0) one can change the value of $\sigma$ to accommodate changes in the disturbance variance and fit different shapes of distribution with respect to one standard.

Taking exponentials at both sides yields an alternative expression:

$$
T_{i}=\exp \left\{\beta_{0}+\beta_{1} x_{i 1}+\ldots+\beta_{k} \mathrm{x}_{\mathrm{ik}}+\sigma \varepsilon_{i}\right\} \text { (A-1) }
$$

When $\sigma>1$, the hazard rate decreaseed with time.

This may be applied to HCC cases treated by hepatic surgical resection.

When $0.5<\sigma<1$, the hazard is increasing but at a decreasing rate. When $0<\sigma<0.5$, the hazard is increasing at an increasing rate.

Let the risk score for small HCC cases treated by PEI be denoted by $R$, defined as follows.

$$
\mathrm{R}=\beta_{0}+\beta_{1} \mathrm{x}_{1}+\ldots+\beta_{\mathrm{k}} \mathrm{x}_{\mathrm{k}}(\mathrm{A}-2\}
$$

Both may be appropriate for HCC cases treated by PEI with TAE or without TAE.

If $T=t$ then the survival function (probability of surviving to time $t$ )for the Weibull model is:

$$
S(t)=\exp \left\{-\left[t e^{-\mathrm{R}}\right]^{1 / \sigma}(\mathrm{A}-3)\right.
$$

\section{References}

[1] Kotoh K, Sakai H, Sakamoto S, et al. The effect of percutaneous ethanol injection therapy of hepatocellular carcinoma is comparable to that of hepatectomy. Am J Gastroenterol 1994;89:194-8.

[2] Shiina S, Tagawa K, Hiwa Y, Unuma T, Komatsu Y, Yoshiura K. Percutaneous ethanol injection therapy for hepatocellular carcinoma:results in 146 patients. American Journal of Roentgenology 1993;160: 1023-8.

[3] Castells A, Bruix J, Bru C, Fuster J. Treatment of small hepatocellular carcinoma in cirrhotic patients: a cohort study comparing surgical resection and percutaneous ethanol injection. Hepatology 1993;18:1121-6.

[4] Livraghi T, Giorgio A, Marin G, et al. Hepatocellular carcinoma and cirrhosis in 746 patients: long-term results of percutaneous ethanol injection. Radiology 1995;197:101-8.

[5] Lencioni R, Bartolozzi C, Caramella D, et al. Treatment of small hepatocellular carcinoma with percutaneous ethanol injection analysis of prognostic factors in 105 western patients. Cancer 1995;76:1737-46.

[6] Pompilli M, Rapaccini GL, de Luca F, et al. Risk factors for intrahepatic recurrence of hepatocellular carcinoma in cirrhotic patients treated by percutaneous ethanol injection. Cancer 1997;79:1501-8.

[7] Ishii H, Okada S, Nose H, Okusaka T, Nagahama H, Nakayama H. Predictive factors for recurrence after percutaneous ethanol injection for solitary hepatocellular carcinoma. Hepato-Gastroenterol 1996;43: 938-43.

[8] Ishii H, Okada S, Nose H, Okusaka T, Yoshimori M, Takayama T. Local recurrence of hepatocellular carcinoma after percutaneous ethanol injection. Cancer 1996;77:1792-6.

[9] Ohnishi K, Tanabe Y, Ryu M, Isono K, Yamamoto Y, Usui S. Prognosis of hepatocellular carcinoma smaller than $5 \mathrm{~cm}$ in relation to treatment: study of 100 patients. Hepatology 1989;7:1285-90.

[10] Chen MF, Hwang TL, Jeng LB, Wang CS, Jan YY, Chen SC. Postoperative recurrence of hepatocellular carcinoma: two hundred five consecutive patients who underwent hepatic resection in 15 years. Arch Surg 1994;129:738-42.

[11] Chen MF, Hwang TL, Jeng LBB, Jan YY, Wang CS, Chou FF. He- 
patic resection in 120 patients with hepatocellular carcinoma. Arch Surg 1989;124:1025-8.

[12] Iwatstuki S, Starzl TE. Personal experience with 411 hepatic resections. Ann Surg 1988;208:421-34

[13] Nagorney DM, van Heerden JA, Ilstrup DM, Adson MA. Primary hepatic malignancy: surgical management and determinants of survival. Surgery 1989;106:740-9.

[14] Tsuzuki T, Sugioka A, Ueda M, et al. Hepatic resection for hepatocellular carcinoma. Surgery 1990;107:511-20.

[15] Collett D. Modelling survival data in medical research. London: Chapman \& Hall; 1994.
[16] Beasley RP, Hwang LY, Lin CC, Chien CS. Hepatocellular carcinoma and hepatitis B virus. A prospective study of 22707 men in Taiwan. Lancet 1981;2:1129-33.

[17] Saito I, Miyamura T, Ohbayashi A, et al. Hepatitis C virus infection is associated with the development of hepatocellular carcinoma. Proc Natl Acad Sci USA 1990;87:6547-9.

[18] Okada S, Sato T, Okusaka T, et al. Past exposure to hepatitis B virus as a risk factor for hepatocellular carcinoma in patients with chronic liver disease. Br J Cancer 1998;77:2028-31.

[19] Allison PD. Survival analysis using the SAS system: a practical guide. Cary, NC: SAS Institute Inc.; 1995. p. 68-9. 